\title{
The Distribution of Nerves in Human Primary Teeth ${ }^{1}$
}

\author{
ROBERT RAPP, ${ }^{2}$ JAMES K. AVERY AND DONALD S. STRACHAN \\ Departments of Pedodontics and Oral Biology, \\ School of Dentistry and The Department of Anatomy, \\ Medical School, The University of Michigan
}

\begin{abstract}
Human primary teeth in the stages of late root formation, root completion and root resorption were impregnated with silver to allow study of the distribution of nerves in the pulp.

Neural trunks composed of many individual, myelinated nerve fibers enter the apical foramen of primary teeth and pass in a coronal direction, often in close association with blood vessels. Neural trunks in the crowns of the teeth divide into smaller branches, which divide further into myelinated nerve fibers, which become interwoven to form the parietal layer (plexus of Raschkow). An occasional unmyelinated fiber leaves this zone and passes into the odontoblastic layer of cells where it appears to terminate with no specialized ending apparent. No nerves were seen to enter the predentin or dentin.

As primary teeth begin to undergo root resorption, degenerative changes such as thickenings, varicosities and fragmentation appear in the nerves. The quantity of neural tissue also decreases. The greater the amount of resorption, the greater are the degenerative changes. In teeth in which the roots are almost completely resorbed, only a small number of nerves remain.

The pattern of distribution of nerves in primary teeth is observed to be similar to that of permanent teeth. The density of the innervation of the primary teeth, however, is not found to be as great as that of permanent teeth.
\end{abstract}

The innervation of human primary teeth has received little attention when compared to similar research on permanent teeth. The lack of interest in the distribution of pulpal nerves in the primary dentition may result from the belief that the pulps are similar to those of permanent teeth or that primary teeth are not a lasting component of the oral cavity. Clinicians have long been aware of a lower sensitivity to pain in primary teeth than in permanent teeth which may be due to differences in number and/or distribution of their neural components. Another area in which information is lacking involves changes in the neural tissue of the primary teeth during the period of root resorption (and exfoliation). The present investigation is concerned, therefore, with the distribution of nerves in primary teeth during stages of root formation, root completion, and root resorption.

Bradlaw ('36) studied neural degenerative changes in both human and animal primary teeth using a variety of silver impregnation techniques. He observed these changes to occur prior to exfoliation in some teeth but not in others. Mohuiddin
('50) studied the primary and permanent teeth of the cat by means of both the Romanes' and Bielschowsky silver methods. The permanent and nonresorbing primary teeth exhibited patterns of innervation similar to those observed for human permanent teeth by Rapp, Avery and Rector ('51). All of these investigators observed neural fibers within the pulp to form arborizations or a parietal layer (plexus of Raschkow) adjacent to the dentin and then terminate between or among the odontoblasts. A few nerves were found in the predentin and dentin, but none were observed coursing within the dentinal tubules. Mohuiddin was interested in whether neural changes preceded or followed the onset of pulpal disorganization during exfoliation. He found degenerative neural changes appear prior to evidence of root resorption. He noted also that degenerative changes were limited to nerves $1 \mu$ or

1 This study was supported in part by U.S.P.H.S. reasearch grant DE 01604 from the National Institute of Dental Research, National Institutes of Health, Bethesda, Maryland and grant 695 of the H. Rackham Fund of the University of Michigan.

2 Present address: Department of Pedodontics, School of Dentistry, University of Pittsburgh, Pittsburgh, Pennsylvania 15213. 
more in diameter. These changes were characterized by varicosities and vesicular formations and fragmentation within the nerve fibers. They were not observed in every nerve fiber or in every fiber within a nerve bundle. As resorption advanced, however, the number of neural fibers in the pulp decreased until only occasional fragments of axons of a fine caliber could be seen.

Fernhead and Linder ('56), and Fernhead ('61) used silver impregnation techniques to study developing and resorbing human primary teeth. Fernhead noted a marked increase of small diameter nerve fibers in the pulps during root formation. The plexiform arrangement of fibers was not evident in the primary teeth at as early a stage as in the permanent teeth. His observations of the organization of neural fibers in permanent teeth correspond to those described by Rapp, Avery and Rector ('57). Fernhead observed a marginal plexus of fibers adjacent to the predentin, from which individual fibers became embedded in the predentin while others entered the dentinal tubules and were closely associated with the odontoblastic processes. He found some nerve fibers to have undergone degenerative changes, in both deciduous and permanent teeth although the latter showed no evidence of root resorption. He concluded that degenerating nerve fibers can usually be found in groups of otherwise normal preterminal nerves and that there is thus no evidence that primary tooth root resorption is under nervous control.

\section{MATERIALS AND METHODS}

Seventy-five human primary teeth in the stages of root formation, root completion and various stages of root resorption were studied. Immediate fixation was carried out in a $10 \%$ solution of neutral formalin containing $10 \%$ chloral hydrate followed by decalcification in $3 \%$ hydrochloric acid. The teeth were embedded in paraffin and sectioned at thicknesses ranging from 10-30 $\mu$. Power's ('52) modification of the Romanes' silver impregnation technique was used to demonstrate neural tissue.

\section{RESULTS}

\section{A. Primary teeth with completed or nearly completed roots}

Nerve trunks containing many individual, myelinated nerve fibers pass through the apical foramen of the tooth into the root canal (fig. 1). Within the root canal, only an occasional branch rises from these trunks, (fig. 2). Many of the nerve trunks within the root canal were found to exist independently of vessels (fig. 1), while others were found in close association with blood vessels (figs. 2,3 ).

The nerve trunks pass into the coronal pulp chamber and then divide into smaller branches, which divide further as they pass laterally towards the dentin walls and into the pulpal horns (fig. 4). These smaller nerve fibers divide and eventually become single fibers which interweave to form the parietal network of nerves (plexus of Raschkow), (figs. 5,6). This plexus, located along the periphery of the pulp chamber adjacent to the cell-rich zone, is composed of both myelinated and unmyclinated fibers (figs. 5, 6). An occasional single, unmyelinated fibers arises from the parietal plexus and passes through the cell-rich zone and cell-free zone (Zone of Weil) to terminate among the odontoblast cells, (figs. 6, 7). No speialized endings or attachments are observed between nerve fibers and the odontoblasts. Nerves are not observed to penetrate the substance of the predentin or dentin. The pattern of distribution of neural elements in the forming and completed primary teeth appears to be very similar to that of young permanent teeth.

\section{B. Primary teeth with resorbing roots}

Primary teeth that have undergone early stages of root resorption display little, if any, change in the pattern of neural distribution from that seen in the nonresorbed teeth. A few signs of neural degeneration in the form of varicosities and vacuoles are seen in a few of the nerve fibers (figs. 8, 9, 10). In teeth with approxmately half of the root dentin resorbed, however, the majority of the fibers exhibit varicosities and vacuole formation. In addition, fragmentation of some of the nerve fibers is observed, as well as a general 
decrease in the total amount of neural tissue present within the tooth (fig. 10). Some teeth show progressive neural degeneration with progressive root resorption while others show little correlation between the amount of neural resorption and degeneration. Teeth with only the crown dentin remaining exhibit a varied pattern of innervation of the pulp organ, (figs. 11, 12, 13). In some instances, a nerve trunk persists in which there is loss of continuity of the fibers (fig. 11). The number of individual fibers within the trunk appears to be decreased in some instances when compared to those seen before onset of root resorption (fig. 13). In other teeth, occasional nerve fibers or groups of fibers or the remnants of a parietal plexus of nerves are seen (fig, 12). On the other hand, some completcly resorbed teeth show a complete absence of nerves.

Root resorption is seen often to begin on the sides of the root rather than at the apical end (fig. 14). In such instances, signs of inflammation may be visible within the pulpal tissue opposite the resorption site. Nerves passing through this area persist, in spite of the resorptive and inflammatory process. Teeth with nearly completely resorbed roots exhibit similar signs of inflammation in the pulp.

The character of the pulpal cells appears to change as root resorption becomes advanced, in that they become either further separated or decreased in number and assume an embryonic-like appearance (fig. 13). The general appearance of the pulp, in addition, takes on a more embryonic characteristic in that the number of silver-impregnated connective tissue fibers, along with nerve fibers, appears to decrease. Multinucleated giant cells are observed frequently to exist in clusters along the predentin border of the teeth, in advanced states of root resorption.

\section{DISCUSSION}

Fernhead ('61) noted an increase in neural components between onset of dentinogenesis and completion of root development. He observed, further, that a plexus of Raschkow is not present in primary teeth by the time of roat completion. This observation is substantiated neither by Mohuiddin ('50) nor by this present study in which the plexus structure is seen during the root completion phase of development. Teeth in the earlier stages of root formation were not included in this investigation, however. Fernhead stated further that it is impossible to compare patterns and density of innervation of primary and permanent teeth at similar stages of development without taking into consideration the fact that permanent teeth require six to nine years between dentinogenesis and root completion as compared to $12-16$ months for primary teeth. This difference in maturation time between primary and permanent teeth does not mean that a primary tooth at root completion cannot be innervated to the same or similar extent as a permanent tooth at a similar stage of development. Evidence of this is supplied by the observation in this study, that a similar pattern of innervation existed at similar stages of development in the primary and permanent teeth.

There appears to be agreement by Mohuiddin ('50), Fernhead ('61) and Rapp, Avery and Rector ('57) that' the general pattern of innervation of both primary and permanent teeth after root resorption has begun is similar. In no instance in the present study, however, were nerves observed to enter the calcified tissues of primary teeth. Numerous authors, on the other hand, have noted neural fibers in predentin and dentin of permanent teeth. Mohuiddin described some nerves passing into the predentin of the cat while most appear to terminate about the odontablasts. Rapp, Avery and Rector have attributed nerves in the predentin matrix to be a result of entrapment during its deposition. Fernhead described nerve fibers entering dentinal tubules and, recently, several investigators have reported nerve fibrils in the dentin. Hattyasy ('61), and Stella and Fuentes ('61) have described intratubular nerve fibers, by means of the light microscope, and recently Frank ('66) reported the presence of such fibrils in dentin. Arwill ('58), on the other hand, studied the dentin extensively with the electron microscope and found no nerve fibers.

Mohuiddin ('50) observed unusual changes in the nerves of deciduous teeth undergoing root resorption and attributed 
them to be degenerative in nature. Fernhead ('61) was of the opinion that similarappearing changes in nerves were due to incomplete fixation. Changes similar to those described by Mohuiddin were observed in the present study. Thickening of the fibers (varicosities), thinning of these enlarged areas (vaculations) and fragmentation were obscrved. These changes were seen frequently following the onset of root resorption, although rarely before this stage. Similar changes were observed in the teeth of vitamin A-deficient rats by King, Lewinsky and Stewart ('38) and were considered to be evidence of neural degeneration.

Similar-appearing changes in nerves of frog tadpoles have been described by Speidell ('36), of the taste buds of catfish by Torrey ('36) and of nerves of rabbit cutaneous tissue by Wedell and Glees ('41). As such changes within neural tissue were not observed in nonresorbing primary teeth or in permanent teeth, their presence is probably not due to such a factor as incomplete fixation.

The earliest and most frequently observed degenerative change was the thickening of the neural fibers and the production of varicosities. With progressive root resorption, formation of vesicles and fragmentation of the fibers were seen. These changes led to a general reduction in total neural density. Degenerative changes, however, were not found in all fibers of the pulp organ or in all fibers within a single neural trunk.

Mohuiddin ('50) described degenerative changes that began in pulpal nerves before the onset of root resorption. The observations of this present study would suggest that such changes follow and occur as a result of onset of root resorption. Observations of this nature must be speculative as it is difficult to determine the exact time of initiation of resorption. Again, many subtle biochemical, if not morphologic, changes may be occurring in the pulp prior to noticeable alteration of the root surface. The observations in this study of a reduction in the amount of neural fibers in the human pulp organ occurring with progressive root resorption are in accordance with the findings of Mohuiddin.
The pattern of distribution of nerves in the nonresorbing, human, primary tooth is similar to that described in the permanent tooth by Rapp, Avery and Rector ('57). The appearance in the primary tooth of the large neural trunks in the central pulp region and the peripheral layer are a common characteristic. The density of the innervation of the primary tooth, however, is not as great as that of the permanent tooth. The plexus of Raschkow of the primary tooth is not composed of as dense a network of myelinated fibers as is the counterpart in the permanent dentition. Fewer fibers are seen to arise, in addition, from this neural network to pass to the odontoblasts in primary teeth when compared to permanent teeth. This reduction in innervation is possibly the reason that primary teeth are less sensitive to operative procedures than are permanent teeth. The presence of giant cells in the odontoblastic layer, as observed by Akai ('65), would agree with the findings of multinucleated giant cells along the predentin border seen in this study. Akai reported, in addition, that collagen fibers in similarly resorbing teeth change to argyrophilic fibrils. The observations of the present study are not of an increase in argyophilic fibers but of a thinning out of the entire pulpal tissue components, be it cells, nerves or connective tissue fibers. Surprisingly, signs of inflammation in the pulps of resorbing teeth are observed in the present study but were not seen by Akai. Neither is oral epithelium observed to infiltrate the pulp as was described by Akai.

It would appear from the observations of the pulps of progressively resorbing teeth that a decrease in complexity (number of pulpal cells, fibers) and the appearance of giant cells indicates that the course of the pulp is that of increasing degeneration.

\section{IITERATURE CITED}

Akai, M., et al. 1965 Changes of blood vessels and connective tissue fibers during shedding of deciduous teeth in dogs. J. Osaka Univ. Dent. Soc., 10; 195-201.

Arwill, Torre 1958 Innervation of the teeth. A study by light and electron microscope. Trans. Royal Sch. Dent., Stockholm and Umea, Series., 2: 3.

Bernick, Sol. 1964 Differences in nerve distribution between erupted and non-erupted human teeth. J. Dent. Res., 43: 406-411. 
Bradlaw, R. 1936 The innervation of teeth. Proc. Royal Soc. Med., 29: 507-508.

Fernhead, R. W. 1961 The neurohistology of human dentine. Proc. Royal Soc. Med., (Odontology), 54: 877-884.

Fernhead, R. W., and J. E. Linder 1956 Observations on the silver impregnation of nerve fibers in teeth. J. Anat, (London), 90: 228-235.

Frank, R. 1966 Ultrastructure of human dentin. Third European Symposium of Calcified Tissues: 259-272. H. Fleisch, H. J. Blackwood, and M. Owen, eds. Springer-Verlag, New York.

Hattyasy, D. 1961 Continuous regeneration of dentinal nerve-endings. Nature, (London), 189: $72-74$.

King, J. D., W. Lewinsky and D. Stewart 1942 Degenerative changes in the axis cylinders of the dental nerves due to diet deficient in vitamin $A$ and carotene. J. Physiol., 93: 206-214.
Mohuiddin, A. 1950 The fate of the nerves of the deciduous teeth. J. Anat., 84: 319-323.

Rapp, Robert, J. K. Avery and R. A. Rector 1957 A study of the distribution of nerves in human teeth. J. Canad. Dent. Assoc., 23: 447-453.

Speidell, C. C. 1936 Studies of living nerves. V. Alcoholic neuritis and recovery. J. Comp. Neur., 64: 77-114.

Stella, A., and A. Fuentes 1961 Inervaion dentinaria intra-canicular. Su demonstracion po rel metodo de la hematoxilina-ferrica de Heidenhain. Anales de la Fac. de Odont. (Montevideo), Supplement to $10: 157$.

Torrey, T. W. 1936 The relation of nerves to degenerating taste buds. J. Comp. Neur., 64: 325-336.

Weddell, G., and P. Glees 1941 The early stages of the degeneration of cutaneous nerve fibers. J. Anat., 76: 65-92. 
The following photomicrographs illustrate sections of human primary cuspids and molars impregnated with silver to delineate neural tissue. Figures 1-8 show teeth in which no root resorption is occurring while figures 9-14 show teeth undergoing root resorption.

\section{Abbreviations}

B V, blood vessels

C F, cell free zone (Zone of Weil)

C. $\mathbf{R}$, cell rich zone

$\mathrm{D}$, dentin

I C, inflammatory cells

$\mathrm{N}$, nerve
$\mathrm{N} \mathbf{N}$, parietal network of nerves (Plexus of Raschkow)

N T, nerve trunks

$O$, odontoblasts

$P$, pulp

$\mathbf{R} \mathbf{R}$, root resorption

PLATE 1

\section{EXPLANATION OF FIGURES}

1 Area of apical foramen of developing primary molar. Several nerve trunks consisting of many myelinated nerve fibers enter the open foramen and pass in a coronal direction. The pulp tissue is highly cellular in this proliferation zone. $\times 67$

2 Midlength region of the root canal of a primary cuspid not undergoing root resorption. Nerve trunks (N T) pass in a coronal direction with a minor amount of branching and often in close association with blood vessels $(B \mathrm{~V}) . \times 33$.

3 Root canal of a completely formed primary cuspid. Many individual, myelinated nerve fibers compose the centrally located nerve trunks. No signs of neural degeneration are evident. 100.

4 Pulp chamber of a nonresorbed primary cuspid. Nerve trunks pass from the root canal into the coronal pulp chamber where they divide into smaller branches. $\times 67$. 

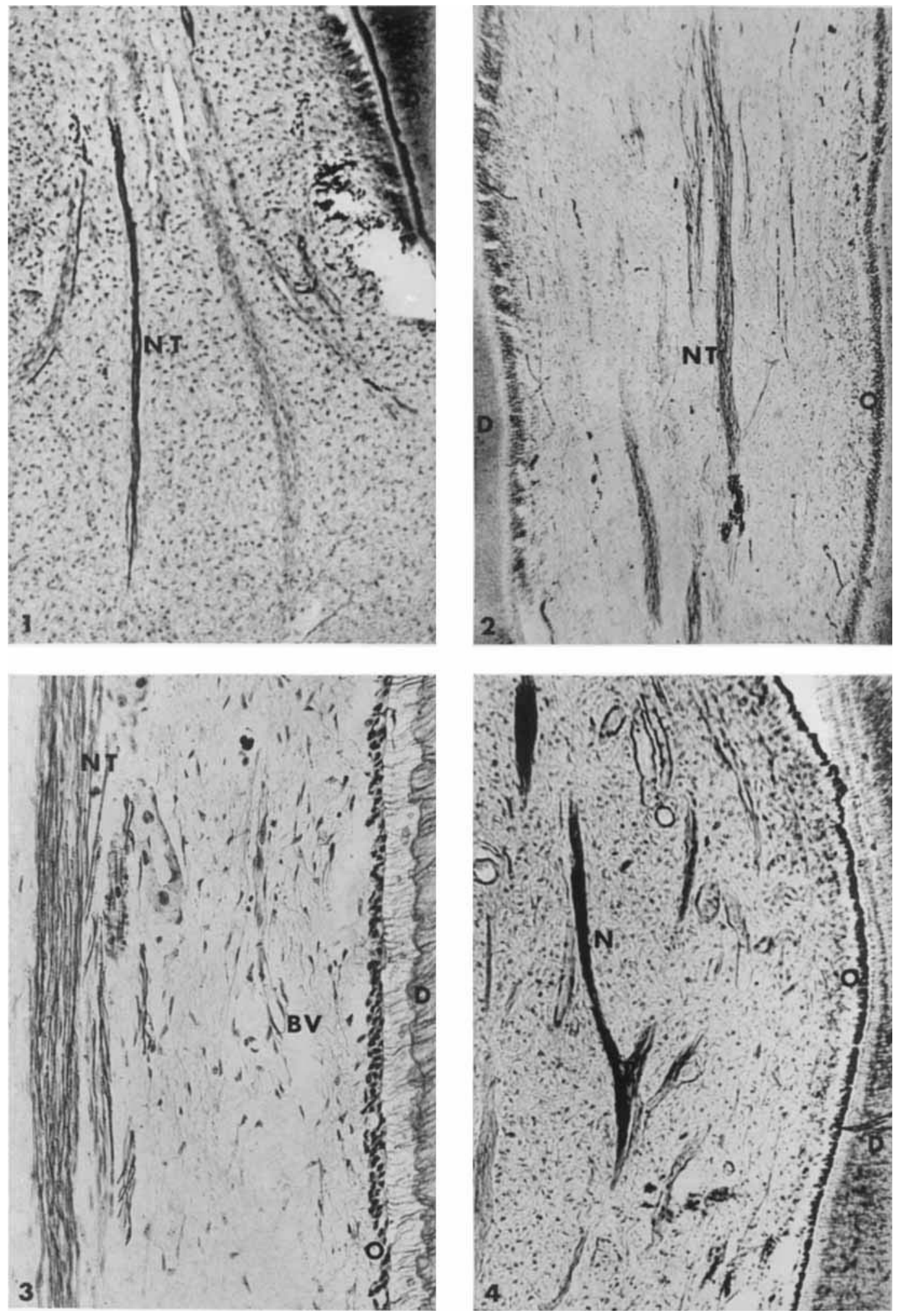
PLATE 2

EXPLANATION OF FIGURES

5 Pulp chamber of a nonresorbed primary cuspid. Individual, myelinated nerve fibers pass peripherally in the pulp organ to form the parietal network of nerves (plexus of Raschkow) (N.N.). Note the cell-rich zone (C R.) adjacent to the neural network. The cell-free zone (C F) lies adjacent to the odontoblasts (O). Many blood vessels containing blood cells are evident in the peripheral area of the pulp. $\times 267$.

6 Pulp chamber of a nonresorbed primary cuspid. Nonmyelinated nerve fibers from the parietal network pass through the cell-rich and cellfree zones to tcrminate without special endings among the odontoblasts. $\times 300$. 

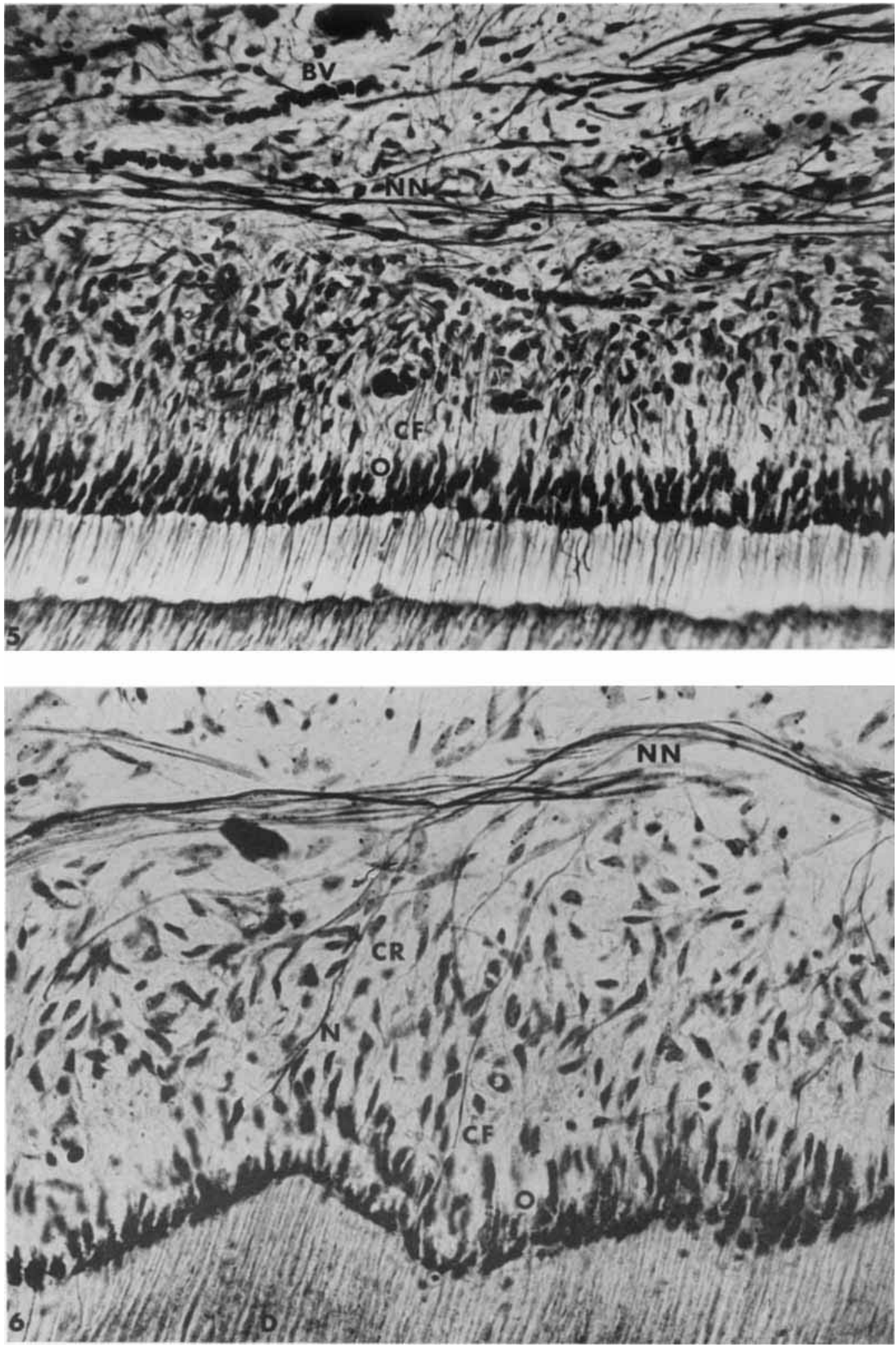


\section{PLATE 3}

EXPLANATION OF FIGURES

7 Pulp chamber of a nonresorbed primary cuspid. A nonmyelinated nerve fiber passes from the parietal network through the cell-rich and cell-free zones to the darkly stained odontoblastic layer $(0)$. $\times 333$.

8 Pulp chamber of a resorbed primary cuspid. A high magnification of a nerve trunk and a blood vessel illustrates the close relationship of the two. Note cvidence of carly degenerative changes in the nerve fibers. $\times 333$. 

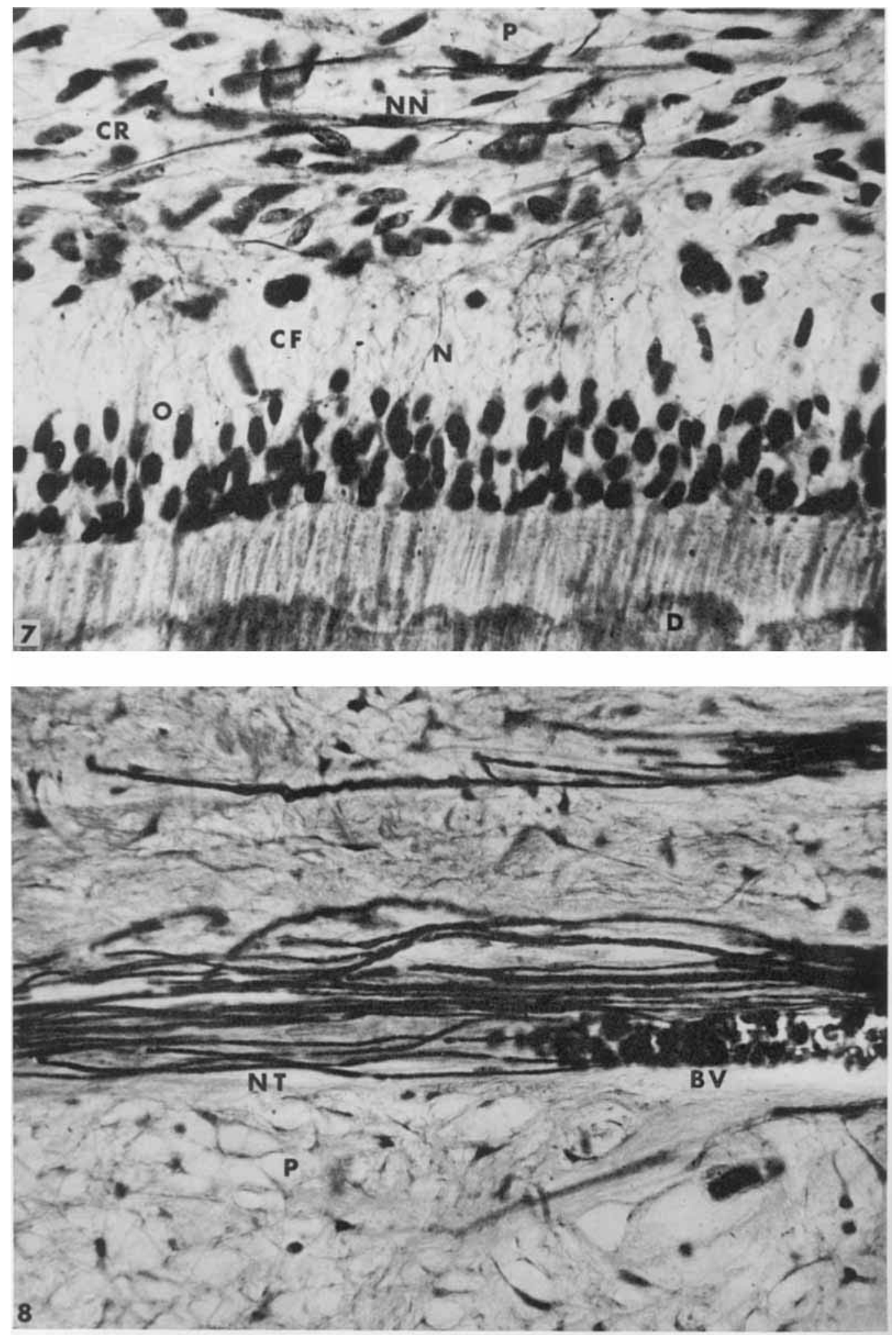
PLATE 4

EXPLANATION OF FIGURES

9 Pulp of a primary cuspid undergoing resorption. A high magnification of nerve branches illustrates degenerative changes which occur upon initiation of root resorption. Varicosities and vacuolation are evident in the nerve fibers. $\times 300$.

10 Pulp of a primary cuspid undergoing resorption. Degenerative changes in the form of varicosities and vacuolation are observed in the nerve tissue. $\times 333$. 

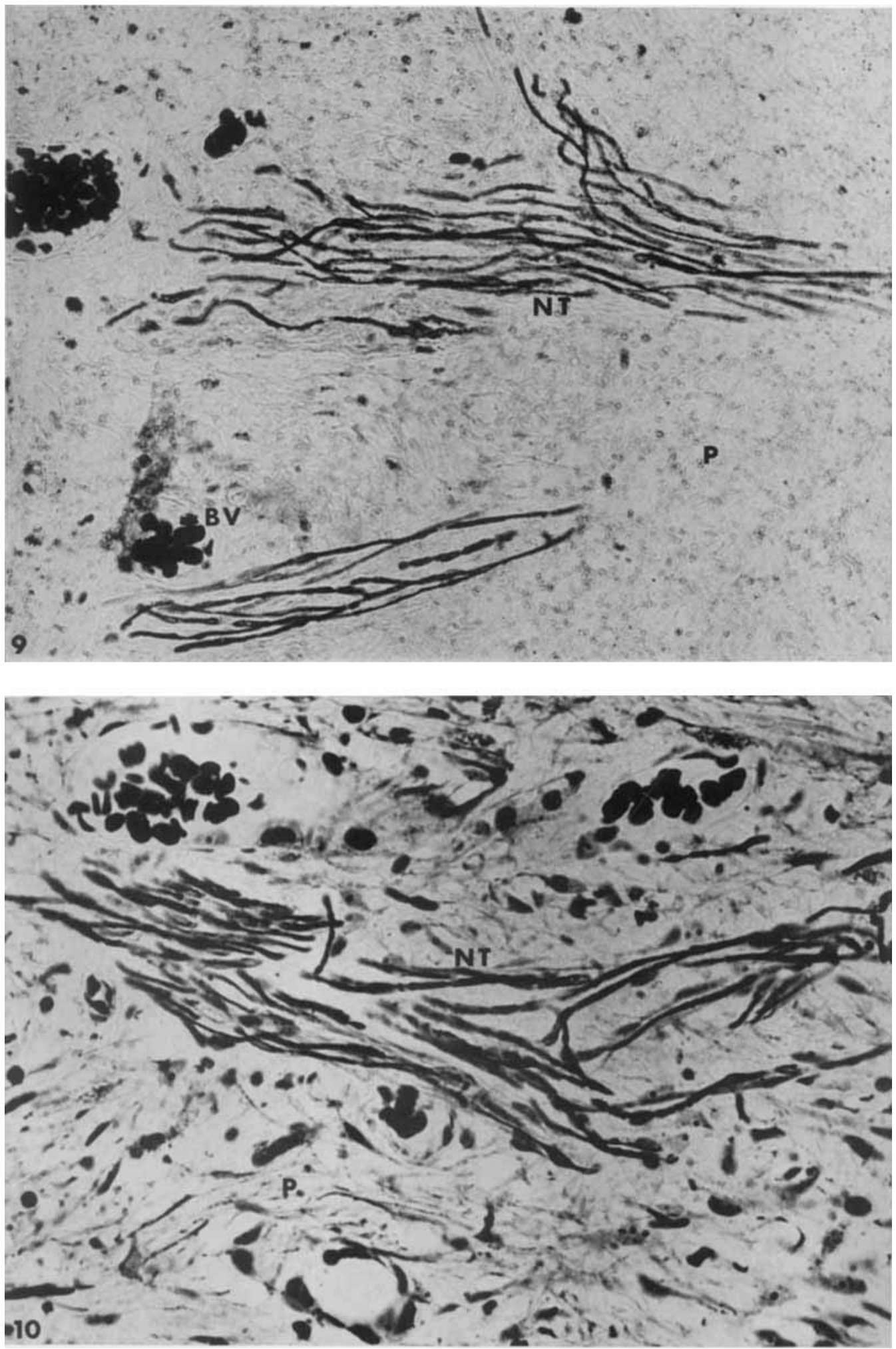
PLATE 5

FXPLANATION OF FIGLRES

11 Pulp chamber of a resorbed primary cuspid. A nerve trunk still per. sists in the pulp following nearly complete resorption of the root dentin. Continuity of the fibers within the trunk appears to be interrupted and the fibers decreased in number. Note the absence of smaller nerve branches or individual fibers. Degenerative signs are present within the nerve tissues as well as evidence of inflammation within the pulp. $\times 83$.

12 Crown of a primary cuspid undergoing root resorption. Several nerve fibers persist although the root dentin of the tooth has been nearly completely resorbed. The oryanization of the nerves seen in nonresorbing teeth is absent and the total neural content decreased. $\times 83$.

13 Crown of a primary cuspid undergoing root resorption. The amount of nerve tissue present in this tooth is less than seen in teeth of figures 9 and 10 although the amount of root resorption is the same. The structure of the pulp tissue is altered; it appears to be more cellular and less fibrous in nature. $\times 20$.

14 Root of a primary cuspid undergoing root resorption. Resorption is taking place along side of the tooth rather than at the apical area. Nerve trunks and branches persist in spite of an inflammatory reaction that appears in response to the process. $\times 20$. 
Robert Rapp, James K. Avery and Donald S. Strachan
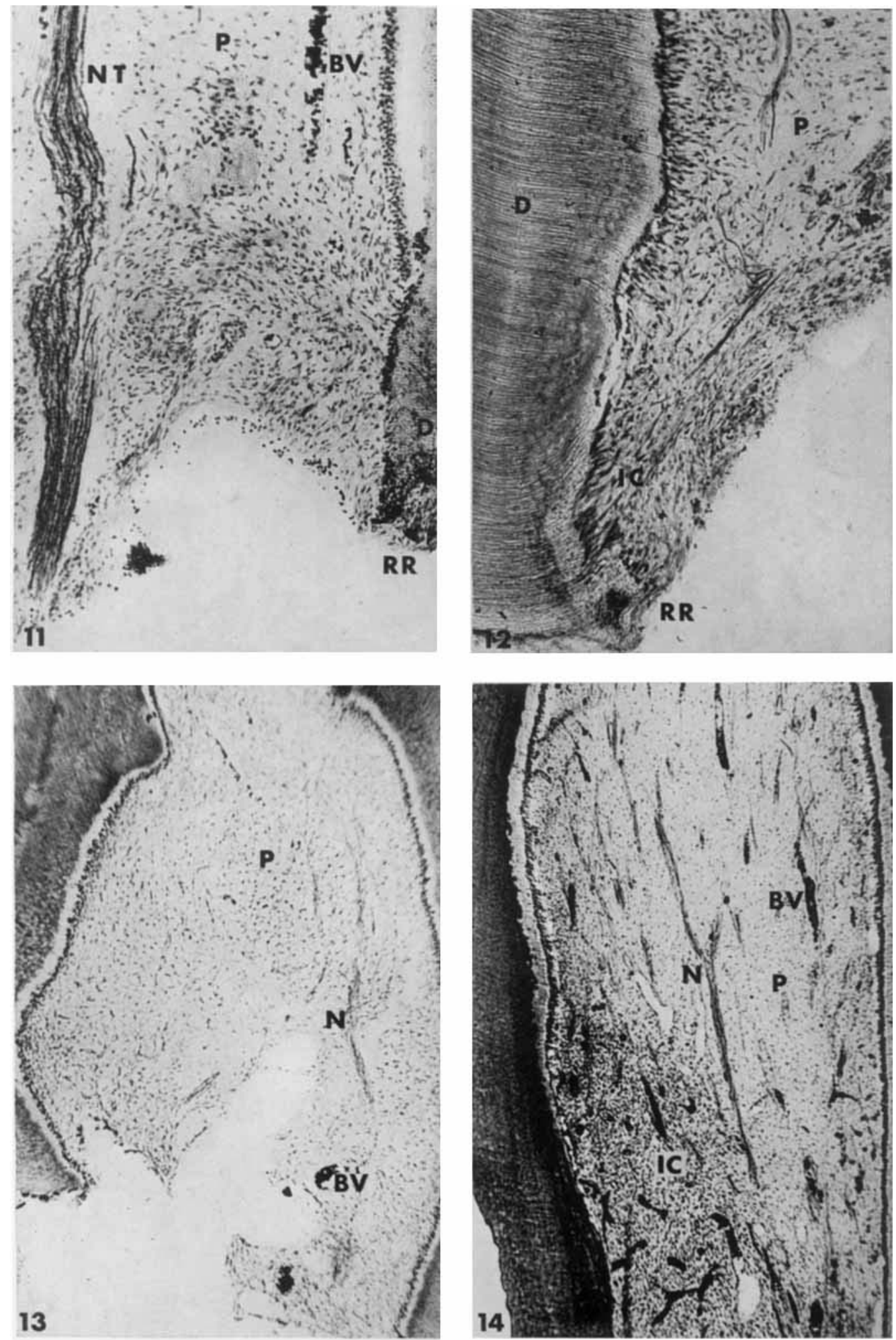